This item was submitted to Loughborough's Research Repository by the author.

Items in Figshare are protected by copyright, with all rights reserved, unless otherwise indicated.

\title{
The thermal boundary layer due to viscous dissipation in impulsively started Poiseuille flow
}

PLEASE CITE THE PUBLISHED VERSION

https://doi.org/10.1093/imamat/hxz001

\section{PUBLISHER}

Oxford University Press on behalf of the Institute of Mathematics and its Applications $(\subset$ The author

\section{VERSION}

AM (Accepted Manuscript)

\section{PUBLISHER STATEMENT}

This is a pre-copyedited, author-produced PDF of an article accepted for publication in IMA Journal of Applied Mathematics following peer review. The version of record KAY, A., 2019. The thermal boundary layer due to viscous dissipation in impulsively started Poiseuille flow. IMA Journal of Applied Mathematics, 84(3), pp. 517532 is available online at: https://academic.oup.com/imamat/article/84/3/517/5301756 and https://doi.org/10.1093/imamat/hxz001.

\section{LICENCE}

CC BY-NC-ND 4.0

\section{REPOSITORY RECORD}

Kay, Anthony. 2019. "The Thermal Boundary Layer Due to Viscous Dissipation in Impulsively Started Poiseuille Flow”. figshare. https://hdl.handle.net/2134/36629. 
IMA Journal of Applied Mathematics (2018) Page 1 of 15

doi:10.1093/imamat/xxx000

\title{
The thermal boundary layer due to viscous dissipation in impulsively started Poiseuille flow
}

\author{
ANTHONY KAY* \\ Department of Mathematical Sciences, Loughborough University, Loughborough, \\ Leicestershire, LE11 $3 T U$ \\ *A.Kay@Lboro.ac.uk
}

[Received on 30 August 2018]

\begin{abstract}
Viscous dissipation occurs in the boundary layers on the walls of a channel in which a flow is accelerated from rest by the sudden imposition of a pressure gradient. We analyse the thermal boundary layer due to this dissipative heating, obtaining numerical solutions and also asymptotic solutions for the cases of both large and small Prandtl number, with both isothermal and adiabatic wall conditions. With large Pr the temperature rise is controlled by the viscous layer, so is independent of Pr and of the wall condition. With small $\mathrm{Pr}$ heat is conducted away from the viscous layer more rapidly, so the temperature rise is reduced as $\operatorname{Pr}$ decreases.
\end{abstract}

Keywords: Viscous dissipation, Boundary layer

\section{Introduction}

Heating by viscous dissipation is often ignored in studies of both free and forced convection. For it to have a significant effect on flow or heat transfer generally requires large length scales and/or large body forces. Nevertheless, it has been suggested that it may be important in rapidly rotating systems (Gebhart, 1962) or in some geophysical applications (Turcotte, Spence and Bau, 1982). In a vertical channel it produces a buoyancy force parallel to the flow (see Barletta (1998) and many subsequent papers by this author and his co-workers), but in a horizontal channel it may cause instability by heating fluid near the lower boundary which therefore becomes less dense than the fluid higher up in the channel (Barletta, 2015). Aside from physical considerations, viscous dissipation yields considerable mathematical interest by introducing a nonlinear dependence of the heat transfer on the flow.

The equations for steady, fully developed forced convection in a vertical channel with isothermal walls admit dual solutions when viscous heating is accounted for: Barletta, Magyari \& Keller (2005) showed that for any specified upward volume flux up to some maximum value, two solutions for the velocity and temperature profiles may be found (although Miklavčič (2015) has shown that one solution branch is unstable). However, no solution exists if the volume flux is greater than the maximum value. The problem was re-formulated by Kay (2016), with the imposed pressure gradient rather than volume flux as the control parameter: dual solutions were found for any given pressure gradient up to a maximum value, with no solution existing for greater values of the forcing. Kay (2016) speculated that imposing a greater upward forcing would yield an unsteady solution with finite-time blow-up: the forced flow would produce high levels of viscous heating, leading to upward buoyancy forces combining with the imposed pressure gradient to produce an ever faster, more strongly heated flow.

To test the blow-up hypothesis will require a numerical solution of the equations for unsteady forced convection. The initial conditions are expected to be of little importance; but some initial conditions do 
need to be specified. The simplest starting point is a static, isothermal condition. The pressure gradient is then imposed from time $t=0$, causing the flow to accelerate, but with boundary layers growing from the no-slip walls until viscous effects are felt throughout the flow. The solution for such a flow without viscous heating was given by Lamb (1927), as a parabolic Poiseuille flow profile plus a transient in the form of a Fourier series. [The solution presented by Lamb was actually for the instantaneous removal of the pressure gradient from an established Poiseuille flow.] Unlike the Fourier series, a numerical scheme might have some difficulty with the discontinuity in the conditions at $t=0$ at the walls. So it would be useful to have an (approximate) analytical solution for the initial growth of the boundary layers, to initiate the numerical solution. We shall see that the buoyancy term in the momentum equation is negligible in this initial stage of the flow, although we shall retain it in our derivation until that fact is established. Thus we shall be considering the forced-convection boundary layer on a plane wall in impulsively started flow with viscous dissipation, which we consider to have its own intrinsic interest even though our original motivation was to overcome an anticipated difficulty in solving the mixed convection problem in a vertical duct.

\section{Governing equations}

We consider two-dimensional, uni-directional, solenoidal flow in a channel of width $2 L$ with vertical, plane, no-slip walls. A constant vertical pressure gradient $\mathrm{d} P_{d} / \mathrm{d} Z$ is imposed, where $P_{d}$ is the difference between total pressure and hydrostatic pressure, and $Z$ is the vertical coordinate, increasing upwards. Under the Boussinesq approximation, the unsteady vertical momentum equation is then

$$
\rho_{m} \frac{\partial W}{\partial t}=-\frac{\mathrm{d} P_{d}}{\mathrm{~d} Z}+\rho_{m} g \beta\left(T-T_{m}\right)+\mu \frac{\partial^{2} W}{\partial Y^{2}},
$$

where $W$ is vertical velocity, $T$ is temperature and $Y$ is the transverse coordinate. The reference temperature $T_{m}$ must be taken to be the cross-section mean of temperature (Barletta \& Zanchini, 1999; Kay, 2016) and may therefore vary in time, but the reference density $\rho_{m}$ is taken to be constant everywhere that it appears explicitly in the equations, under the Boussinesq approximation. The viscosity $\mu$ and thermal expansion coefficient $\beta$ are assumed constant, as are the thermal conductivity $k$ and specific heat $c$ in the energy equation,

$$
\rho_{m} c \frac{\partial T}{\partial t}=k \frac{\partial^{2} T}{\partial Y^{2}}+\mu\left(\frac{\partial W}{\partial Y}\right)^{2} .
$$

Here we have used a formulation which includes viscous heating but not pressure work, which Barletta $(2008,2009)$ argues is consistent with the Boussinesq approximation.

The initial conditions are static, isothermal fluid:

$$
W=0 \quad \text { and } \quad T=T_{0} \quad \text { at } \quad t=0 .
$$

The no-slip condition applies at the walls, and we shall consider both the cases of isothermal and adiabatic walls:

$$
W=0 \quad \text { and either } \quad T=T_{0} \quad \text { or } \quad \frac{\partial T}{\partial Y}=0 \quad \text { at } \quad Y= \pm L .
$$

To define the dimensionless transverse coordinate $y$ and the dimensionless time $\tau$ we use the halfwidth of the channel and a conduction time-scale:

$$
y=\frac{Y}{L}, \quad \tau=\frac{k}{\rho_{m} c L^{2}} t .
$$


However, the scales used for defining the dimensionless velocity $w$ and temperature $\theta$ are derived from a balance between conduction and viscous heating (Miklavčič \& Wang, 2011; Kay, 2016):

$$
w=\frac{\rho_{m} g \beta L^{2}}{k} W, \quad \theta=\frac{\left(\rho_{m} g \beta L^{2}\right)^{2}}{k \mu}\left(T-T_{0}\right) .
$$

A scale for defining the dimensionless pressure gradient $\Pi$ is obtained from a Poiseuille flow balance with our velocity scale:

$$
\Pi=\frac{\rho_{m} g \beta L^{4}}{k \mu} \frac{\mathrm{d} P_{d}}{\mathrm{~d} Z} .
$$

A discussion of physical magnitudes will be postponed until after we have solved the equations; for now, we note that the above definitions yield dimensionless momentum and energy equations,

$$
\begin{aligned}
\frac{1}{\operatorname{Pr}} \frac{\partial w}{\partial \tau} & =-\Pi+\left(\theta-\theta_{m}\right)+\frac{\partial^{2} w}{\partial y^{2}} \\
\frac{\partial \theta}{\partial \tau} & =\frac{\partial^{2} \theta}{\partial y^{2}}+\left(\frac{\partial w}{\partial y}\right)^{2}
\end{aligned}
$$

in which there are only two control parameters, the pressure gradient $\Pi$ and the Prandtl number

$$
\operatorname{Pr}=\frac{c \mu}{k}
$$

The initial conditions are

$$
w=0 \quad \text { and } \quad \theta=0 \quad \text { at } \quad \tau=0
$$

The boundary conditions are

$$
w=0 \quad \text { and either } \quad \theta=0 \quad \text { or } \quad \frac{\partial \theta}{\partial y}=0 \quad \text { at } \quad y= \pm 1
$$

The dimensionless cross-section mean temperature is

$$
\theta_{m}=\frac{1}{2} \int_{-1}^{1} \theta \mathrm{d} y
$$

\section{The momentum boundary layer}

Our concern is with how the flow starts up. The initial conditions satisfy the energy equation (2.9) but not the momentum equation (2.8) when a non-zero pressure gradient $\Pi$ is imposed. However, the buoyancy and viscous terms are zero at $t=0$, so the momentum equation is satisfied by a uniformly accelerating flow,

$$
w=-\operatorname{Pr} \Pi \tau .
$$

This does not satisfy the no-slip condition at the walls, so there are boundary layers in which the viscous term must be included:

$$
\frac{1}{\operatorname{Pr}} \frac{\partial w}{\partial \tau}=-\Pi+\frac{\partial^{2} w}{\partial y^{2}}
$$


Equation (3.2) shows that the boundary layer thickness is $\sim(\operatorname{Pr} \tau)^{1 / 2}$; we therefore define a similarity variable for the flow near the wall $y=1$,

$$
\eta=\frac{1-y}{(2 \operatorname{Pr} \tau)^{1 / 2}}
$$

and we let

$$
w(y, \tau)=-\operatorname{Pr} \Pi \tau v(\eta) .
$$

The flow is symmetric about $y=0$, so we do not need to consider the wall $y=-1$ separately. The continued neglect of the buoyancy term in (2.8) is justified by observing that $\partial w / \partial y=O\left(\tau^{1 / 2}\right)$ in the boundary layer, so (2.9) implies that $\theta=O\left(\tau^{2}\right)$ as $\tau \rightarrow 0$.

In terms of the new variables, the boundary layer equation (3.2) becomes

$$
v^{\prime \prime}+\eta v^{\prime}-2 v=-2
$$

(where primes denote differentiation with respect to $\eta$ ), with

$$
v(0)=0, \quad v \rightarrow 1 \text { as } \eta \rightarrow \infty .
$$

This problem is essentially the same as that solved by Blasius (1908) in his study of the boundary layer on a flat plate in accelerating flow. The solution is

$$
v=-\eta^{2}+\sqrt{\frac{2}{\pi}} \eta \exp \left(-\frac{1}{2} \eta^{2}\right)+\left(1+\eta^{2}\right) \operatorname{erf}\left(\frac{\eta}{\sqrt{2}}\right) .
$$

This velocity profile is plotted in Figure 1. Bear in mind that the velocity magnitude increases proportional to $\tau$, while the width scale of the boundary layer increases proportionally to $\tau^{1 / 2}$. The velocity profile remains valid until either the buoyancy force in the momentum equation becomes significant or the width of the boundary layer becomes comparable to the channel width.

\section{The thermal boundary layer: isothermal walls}

According to (3.7), the viscous heating term in the energy equation (2.9) is

$$
\left(\frac{\partial w}{\partial y}\right)^{2}=2 \operatorname{Pr} \Pi^{2} \tau\left\{-\eta \operatorname{erfc}\left(\frac{\eta}{\sqrt{2}}\right)+\sqrt{\frac{2}{\pi}} \exp \left(-\frac{1}{2} \eta^{2}\right)\right\}^{2}
$$

within the momentum boundary layer, and vanishes outside the boundary layer. To balance the viscous heating and temperature change terms in (2.9), we therefore let

$$
\theta(y, \tau)=\operatorname{Pr} \Pi^{2} \tau^{2} \psi(\zeta),
$$

where the similarity variable for the thermal boundary layer near the wall $y=1$ is

$$
\zeta=\frac{1-y}{(2 \tau)^{1 / 2}}=\operatorname{Pr}^{1 / 2} \eta
$$




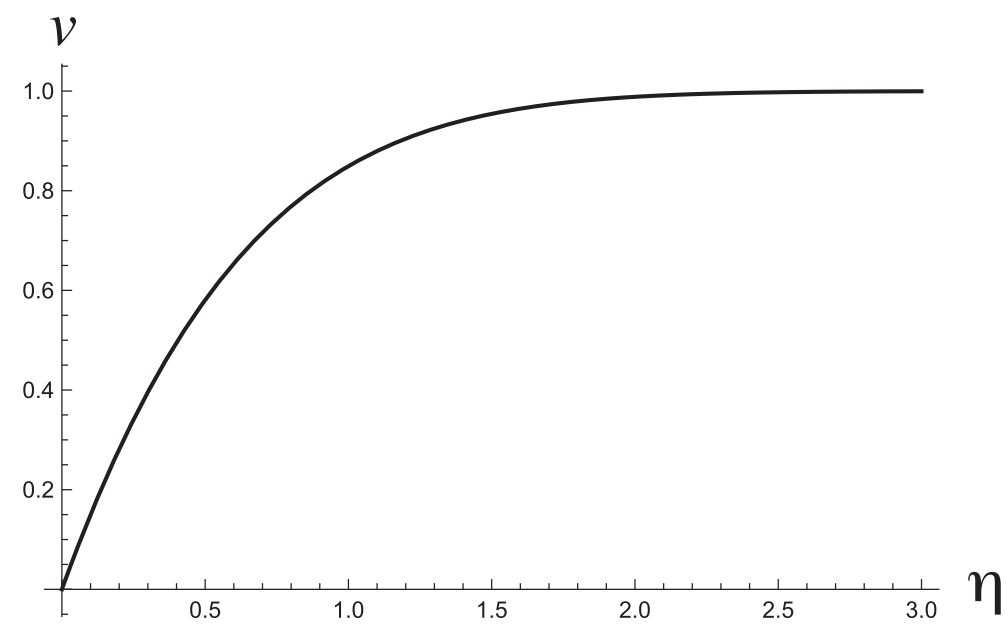

FIG. 1. Velocity profile according to the solution (3.7)

In terms of the new variables, (2.9) becomes

$$
\frac{\mathrm{d}^{2} \psi}{\mathrm{d} \zeta^{2}}+\zeta \frac{\mathrm{d} \psi}{\mathrm{d} \zeta}-4 \psi=-4\left\{-\frac{\zeta}{\operatorname{Pr}^{1 / 2}} \operatorname{erfc}\left(\frac{\zeta}{(2 \operatorname{Pr})^{1 / 2}}\right)+\sqrt{\frac{2}{\pi}} \exp \left(-\frac{\zeta^{2}}{2 \operatorname{Pr}}\right)\right\}^{2} .
$$

With isothermal walls, the boundary conditions are

$$
\psi(0)=0, \quad \psi \rightarrow 0 \text { as } \zeta \rightarrow \infty .
$$

The complementary function for (4.4) is

$$
\psi_{c}=A\left\{3+6 \zeta^{2}+\zeta^{4}\right\}+B\left\{\sqrt{\frac{2}{\pi}}\left(5 \zeta+\zeta^{3}\right) \exp \left(-\frac{1}{2} \zeta^{2}\right)+\left(3+6 \zeta^{2}+\zeta^{4}\right) \operatorname{erf}\left(\frac{\zeta}{\sqrt{2}}\right)\right\}
$$

where $A$ and $B$ are arbitrary constants. A particular integral is available from Mathematica (C)Wolfram Research, Inc.) in closed form, but is too complicated to be of any practical use. So we shall display results of a numerical solution of (4.4) and (4.5) with $\operatorname{Pr}=1$, and then obtain asymptotic solutions in the limits of large and small Prandtl numbers, corresponding respectively to very viscous liquids such as glycerine and liquid metals.

Figure 2 shows the solution with $\operatorname{Pr}=1$. The following numerical data, which relate to the temperature increase in the boundary layer, the wall heat flux and the total rate of energy dissipation, are of interest. The maximum value of $\psi$ is $\psi_{M}=0.07719$, attained at $\zeta_{M}=0.3745$. Furthermore,

$$
\left.\frac{\mathrm{d} \psi}{\mathrm{d} \zeta}\right|_{\zeta=0}=0.5319
$$

and

$$
\int_{0}^{\infty} \psi(\zeta) \mathrm{d} \zeta=0.06988
$$




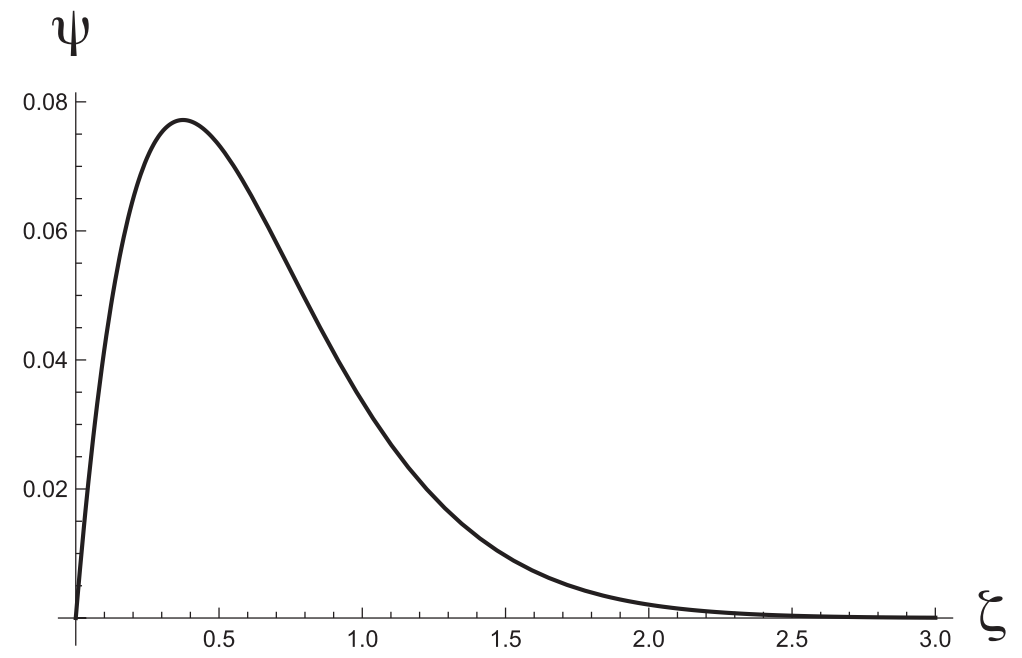

FIG. 2. Temperature profile in the thermal boundary layer for $\operatorname{Pr}=1$, from numerical solution of (4.4) with (4.5).

\subsection{Boundary layer structure at large Prandtl number}

With large Pr there is a relatively broad viscous boundary layer in which the temperature is raised by viscous heating, and the temperature profile adjusts to the wall condition in a narrow thermal boundary layer. Outside the thermal boundary layer, heat conduction is negligible, as can be seen by writing (4.4) in terms of the viscous similarity variable, as

$$
\frac{1}{\operatorname{Pr}} \frac{\mathrm{d}^{2} \psi}{\mathrm{d} \eta^{2}}+\eta \frac{\mathrm{d} \psi}{\mathrm{d} \eta}-4 \psi=-4\left\{-\eta \operatorname{erfc}\left(\frac{\eta}{\sqrt{2}}\right)+\sqrt{\frac{2}{\pi}} \exp \left(-\frac{1}{2} \eta^{2}\right)\right\}^{2} .
$$

If we ignore the conduction (second derivative) term which is $O\left(\operatorname{Pr}^{-1}\right)$ as $\operatorname{Pr} \rightarrow \infty$, we obtain

$$
\frac{\mathrm{d}}{\mathrm{d} \eta}\left(\eta^{-4} \psi\right)=-4 \eta^{-5}\left\{-\eta \operatorname{erfc}\left(\frac{\eta}{\sqrt{2}}\right)+\sqrt{\frac{2}{\pi}} \exp \left(-\frac{1}{2} \eta^{2}\right)\right\}^{2} .
$$

This cannot be integrated in closed form, but the constant of integration (which would multiply $\eta^{4}$ in the solution for $\psi$ ) must be set to zero in order to satisfy the boundary condition as $\eta \rightarrow \infty$. An expansion of the right hand side of (4.10) for large $\eta$ yields

$$
\psi \sim \exp \left(-\eta^{2}\right)\left\{\frac{4}{\pi} \eta^{-6}+O\left(\eta^{-8}\right)\right\} \quad \text { as } \eta \rightarrow \infty,
$$

although increasing numerical coefficients in higher-order terms mean that this approximation is not very useful computationally. Of more interest is the limit of small $\eta$, which is required for matching with the thermal boundary layer: the right hand side of (4.10) is expanded to yield

$$
\frac{\mathrm{d}}{\mathrm{d} \eta}\left(\eta^{-4} \psi\right)=-\frac{8}{\pi} \eta^{-5}+\frac{8 \sqrt{2}}{\sqrt{\pi}} \eta^{-4}+O\left(\eta^{-3}\right)
$$




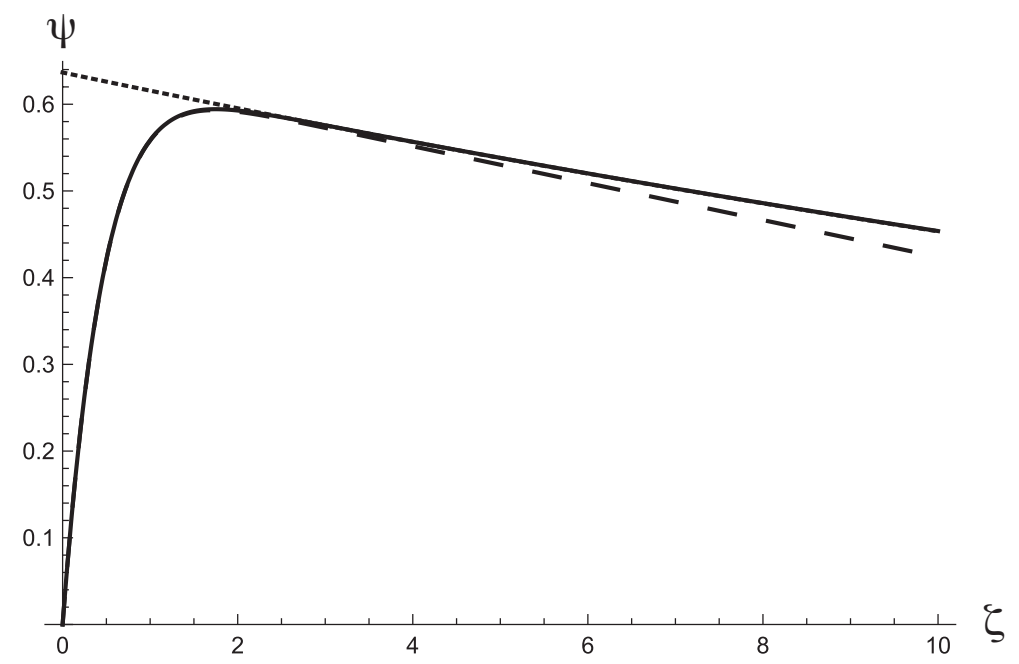

FIG. 3. Temperature profiles in the thermal boundary layer for $\operatorname{Pr}=10^{4}$ with fixed wall temperature: numerical solution of (4.4) with (4.5) (solid line); inner solution (4.15) for large Pr (dashed line); outer (zero-conduction) solution from (4.10) (dotted line).

from which

$$
\psi \sim \frac{2}{\pi}-\frac{8 \sqrt{2}}{3 \sqrt{\pi}} \eta+O\left(\eta^{2}\right) \quad \text { as } \eta \rightarrow 0 .
$$

Further terms in this expansion provide the best means of computing the solution, avoiding the awkwardness for numerical integration of having a removable singularity.

Within the thermal boundary layer, the expansion of the right hand side of (4.4) for large Pr is the same as the expansion of the right hand side of (4.9) for small $\eta$, so the linear approximation to the particular integral of (4.4) is the same as (4.13) (with $\eta=\zeta / \operatorname{Pr}^{1 / 2}$ ). However, we now need to add the complementary function (4.6), which must vanish as $\zeta \rightarrow \infty$ (because the particular integral is equal to the large- $\zeta$ limit of $\psi$ ), yielding $B=-A$ in (4.6). The wall condition $\psi(0)=0$ then gives

$$
A=-\frac{2}{3 \pi} .
$$

Thus the solution valid in the thermal (inner) boundary layer is

$$
\psi=\frac{2}{\pi}-\frac{8 \sqrt{2}}{3 \sqrt{\pi}} \frac{\zeta}{\sqrt{\operatorname{Pr}}}+\frac{2 \sqrt{2}}{3 \pi^{3 / 2}}\left(5 \zeta+\zeta^{3}\right) \exp \left(-\frac{1}{2} \zeta^{2}\right)-\frac{2}{3 \pi}\left(3+6 \zeta^{2}+\zeta^{4}\right) \operatorname{erfc}\left(\frac{\zeta}{\sqrt{2}}\right)
$$

which is compared with the numerical solution of (4.4) with (4.5) for $\operatorname{Pr}=10^{4}$ and with the solution of the zero-conduction equation (4.10) in Figure 3.

Differentiating (4.15), we obtain

$$
\frac{\mathrm{d} \psi}{\mathrm{d} \zeta}=-\frac{8 \sqrt{2}}{3 \sqrt{\pi}} \frac{1}{\sqrt{\operatorname{Pr}}}+\frac{2 \sqrt{2}}{3 \pi^{3 / 2}}\left(8+4 \zeta^{2}\right) \exp \left(-\frac{1}{2} \zeta^{2}\right)-\frac{2}{3 \pi}\left(12 \zeta+4 \zeta^{3}\right) \operatorname{erfc}\left(\frac{\zeta}{\sqrt{2}}\right)
$$


so that

$$
\left.\frac{\mathrm{d} \psi}{\mathrm{d} \zeta}\right|_{\zeta=0}=\frac{16 \sqrt{2}}{3 \pi^{3 / 2}}\left(1-\frac{\pi}{2 \sqrt{\mathrm{Pr}}}\right)
$$

With $\operatorname{Pr}=10^{4}$, this formula from the large-Pr approximation yields the value 1.3332, agreeing to 4 significant figures with a value obtained from the numerical solution of the full boundary-layer equation (4.4). Equation (4.16) does not allow an exact analytical solution for the location $\zeta_{M}$ or magnitude $\psi_{M}$ of the maximum of $\psi$; however, the form of (4.16) suggests $\zeta_{M} \sim \sqrt{\ln (\operatorname{Pr})}$, and indeed numerical calculation yields that

$$
\zeta_{M} \approx \sqrt{\ln (\operatorname{Pr})}-1.31
$$

is a very good approximation to the location of the maximum of the solution (4.15) for $10^{2} \leqslant \operatorname{Pr} \leqslant$ $10^{4}$. Calculations with higher Pr suggest that terms of order $\ln (\ln (\mathrm{Pr}))$ have been omitted; but more importantly, values of $\zeta_{M}$ taken from the numerical solution of the full boundary-layer equation (4.4) are approximated by (4.18) with an error of about 0.13 at $\operatorname{Pr}=10^{2}$, decreasing to less than 0.02 at $\operatorname{Pr}=10^{4}$. An approximation to $\psi_{M}$ may then be found by substituting a value for $\zeta_{M}$ into the linear approximation (4.13) for the outer layer, given the observation from Figure 3 that the outer solution is close to the full solution near the latter's maximum.

\subsection{Boundary layer structure at small Prandtl number}

With small $\mathrm{Pr}$, heat is generated in a thin viscous boundary layer and then conducted outwards into a thicker thermal layer. In the viscous layer, equation (4.9) applies, and we require $\psi=O(\operatorname{Pr})$ in order to balance the conduction term with the viscous heating when $\operatorname{Pr} \ll 1$. So we let

$$
\psi=\operatorname{Pr} \phi
$$

to obtain

$$
\frac{\mathrm{d}^{2} \phi}{\mathrm{d} \eta^{2}}+\operatorname{Pr} \eta \frac{\mathrm{d} \phi}{\mathrm{d} \eta}-4 \operatorname{Pr} \phi=-4\left\{-\eta \operatorname{erfc}\left(\frac{\eta}{\sqrt{2}}\right)+\sqrt{\frac{2}{\pi}} \exp \left(-\frac{1}{2} \eta^{2}\right)\right\}^{2} .
$$

We now ignore the terms of order Pr in (4.20), so that we can simply integrate the right hand side twice and apply the wall boundary condition $\phi(0)=0$ to obtain

$$
\begin{aligned}
\phi=C \eta+ & \frac{4}{\pi}-1-\frac{16}{3 \sqrt{\pi}} \eta \operatorname{erf}(\eta)+\frac{1}{3}\left(3-\eta^{4}\right)\left(\operatorname{erfc}\left(\frac{\eta}{\sqrt{2}}\right)\right)^{2} \\
& +\frac{2 \sqrt{2}}{3 \sqrt{\pi}} \eta\left(\eta^{2}-1\right) e^{-\eta^{2} / 2} \operatorname{erfc}\left(\frac{\eta}{\sqrt{2}}\right)-\frac{2}{3 \pi}\left(6+\eta^{2}\right) e^{-\eta^{2}} .
\end{aligned}
$$

In the outer (thermal) layer, viscous heating is negligible: the right hand side of (4.4) is exponentially small when $\operatorname{Pr}$ is small and $\zeta$ is of order unity. Thus its solution is simply the complementary function (4.6), and the condition that $\psi \rightarrow 0$ as $\zeta \rightarrow \infty$ then gives $B=-A$, so

$$
\psi=A\left\{\left(3+6 \zeta^{2}+\zeta^{4}\right) \operatorname{erfc}\left(\frac{\zeta}{\sqrt{2}}\right)-\sqrt{\frac{2}{\pi}}\left(5 \zeta+\zeta^{3}\right) \exp \left(-\frac{1}{2} \zeta^{2}\right)\right\}
$$




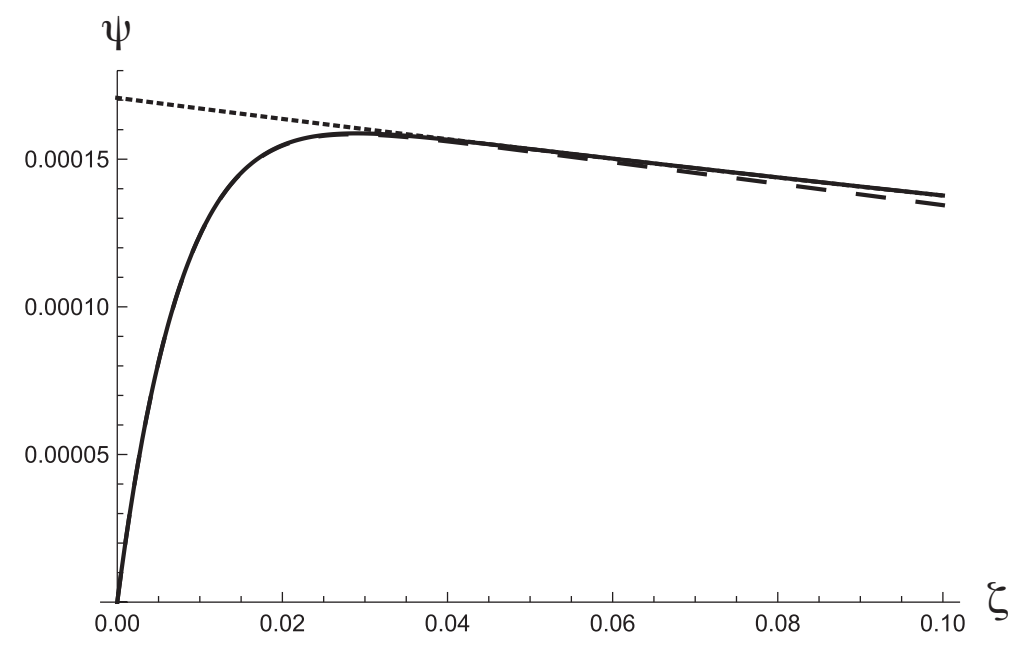

FIG. 4. Temperature profile in the thermal boundary layer for $\operatorname{Pr}=6.25 \times 10^{-4}$, from numerical solution of (4.4) (solid line) and asymptotic solutions for small $\operatorname{Pr}$ : inner solution $\psi=\operatorname{Pr} \phi$ with $\phi$ from (4.21) (dashed line) and outer solution (4.22) (dotted line), with constants given by (4.25).

The constants $A$ and $C$ in (4.21) and (4.22) are determined by the following matching procedure. As $\eta \rightarrow \infty$, the inner solution (4.21) becomes

$$
\begin{aligned}
\phi & \sim C \eta+\frac{4}{\pi}-1-\frac{16}{3 \sqrt{\pi}} \eta+\text { exponentially small terms } \\
\therefore \psi & \sim \operatorname{Pr}\left(\frac{4}{\pi}-1\right)+\operatorname{Pr}^{1 / 2}\left(C-\frac{16}{3 \sqrt{\pi}}\right) \zeta .
\end{aligned}
$$

As $\zeta \rightarrow 0$, the outer solution (4.22) becomes

$$
\psi \sim A\left(3-\frac{8 \sqrt{2}}{\sqrt{\pi}} \zeta\right)+O\left(\zeta^{2}\right)
$$

Equating (4.24) to (4.23) then yields

$$
A=\frac{1}{3}\left(\frac{4}{\pi}-1\right) \operatorname{Pr}, \quad C=\frac{16}{3 \sqrt{\pi}}\left(1-\sqrt{\frac{\operatorname{Pr}}{2}}\left(\frac{4}{\pi}-1\right)\right) .
$$

The numerical solution of the full boundary layer equation (4.4) is plotted for $\operatorname{Pr}=6.25 \times 10^{-4}$ in Figure 4, together with the inner and outer asymptotic solutions.

From the inner solution (4.21) we find

$$
\left.\frac{\mathrm{d} \psi}{\mathrm{d} \zeta}\right|_{\zeta=0}=\frac{8 \sqrt{2}}{3 \sqrt{\pi}}\left\{(\sqrt{2}-1) \operatorname{Pr}^{1 / 2}+\left(1-\frac{4}{\pi}\right) \operatorname{Pr}\right\}
$$


in good agreement with values obtained from the numerical solution of (4.4) (difference of less than $0.5 \%$ at $\operatorname{Pr}=0.01)$. The location and magnitude of the maximum of temperature are controlled by the inner, viscous layer, so $\zeta_{M} \propto \operatorname{Pr}^{1 / 2}$ (or $\eta_{M} \sim$ constant) and $\psi_{M} \propto \operatorname{Pr}$; furthermore, $\int_{0}^{\infty} \psi(\zeta) \mathrm{d} \zeta \propto \operatorname{Pr}$, since the height of the $\psi$ profile is proportional to $\operatorname{Pr}$ and its width is constant (on the $\zeta$ scale). These theoretical scalings are borne out by computations with several small values of Prandtl number.

\section{The thermal boundary layer: adiabatic walls}

The thermal boundary layer equation is still (4.4), but the boundary conditions are now

$$
\psi^{\prime}(0)=0, \quad \psi \rightarrow 0 \text { as } \zeta \rightarrow \infty .
$$

With these boundary conditions, an integration of (4.4) yields

$$
\begin{aligned}
\int_{0}^{\infty} \psi \mathrm{d} \zeta & =\frac{4}{5} \int_{0}^{\infty}\left\{-\frac{\zeta}{\operatorname{Pr}^{1 / 2}} \operatorname{erfc}\left(\frac{\zeta}{(2 \operatorname{Pr})^{1 / 2}}\right)+\sqrt{\frac{2}{\pi}} \exp \left(-\frac{\zeta^{2}}{2 \operatorname{Pr}}\right)\right\}^{2} \mathrm{~d} \zeta \\
& =\frac{16-8 \sqrt{2}}{15 \sqrt{\pi}} \operatorname{Pr}^{1 / 2} \approx 0.17626 \operatorname{Pr}^{1 / 2} .
\end{aligned}
$$

This is simply the specialisation to our boundary-layer flow of the equation

$$
\rho_{m} c \frac{\mathrm{d}}{\mathrm{d} t} \int_{0}^{\infty} T \mathrm{~d} y=\mu \int_{0}^{\infty}\left(\frac{\partial W}{\partial Y}\right)^{2} \mathrm{~d} y
$$

which is obtained by integrating (2.2) with an adiabatic boundary condition at $y=0$, and states that any heat generated by viscous dissipation in the boundary layer must remain within the fluid.

The numerical solution of (4.4) with (5.1) in the case $\operatorname{Pr}=1$ is plotted in figure 5. The dimensionless temperature at the wall in this case is $\psi(0)=0.2500$.

\subsection{Boundary layer structure at large Prandtl number}

The solution in this case proceeds as in Section 4.1 above until the wall condition is applied. Using $\psi^{\prime}(0)=0$ yields the coefficient in the complementary function in the inner solution as

$$
A=-\frac{1}{3 \operatorname{Pr}^{1 / 2}}
$$

instead of (4.14), so that

$$
\psi=\frac{2}{\pi}-\frac{8 \sqrt{2}}{3 \sqrt{\pi}} \frac{\zeta}{\sqrt{\operatorname{Pr}}}+\frac{\sqrt{2}}{3 \sqrt{\pi \operatorname{Pr}}}\left(5 \zeta+\zeta^{3}\right) \exp \left(-\frac{1}{2} \zeta^{2}\right)-\frac{1}{3 \sqrt{\operatorname{Pr}}}\left(3+6 \zeta^{2}+\zeta^{4}\right) \operatorname{erfc}\left(\frac{\zeta}{\sqrt{2}}\right)
$$

which is compared with the numerical solution of (4.4) with (5.1) for $\operatorname{Pr}=10^{4}$ and with the solution of the zero-conduction equation (4.10) in Figure 6. The wall temperature according to (5.5) is

$$
\psi(0)=\frac{2}{\pi}-\frac{1}{\sqrt{\operatorname{Pr}}},
$$

which agrees fairly well with the result of the numerical solution: at $\operatorname{Pr}=10^{4}$, the value of $2 / \pi-\psi(0)$ from the numerical solution is 0.00984 as compared with 0.01 according to (5.6). 


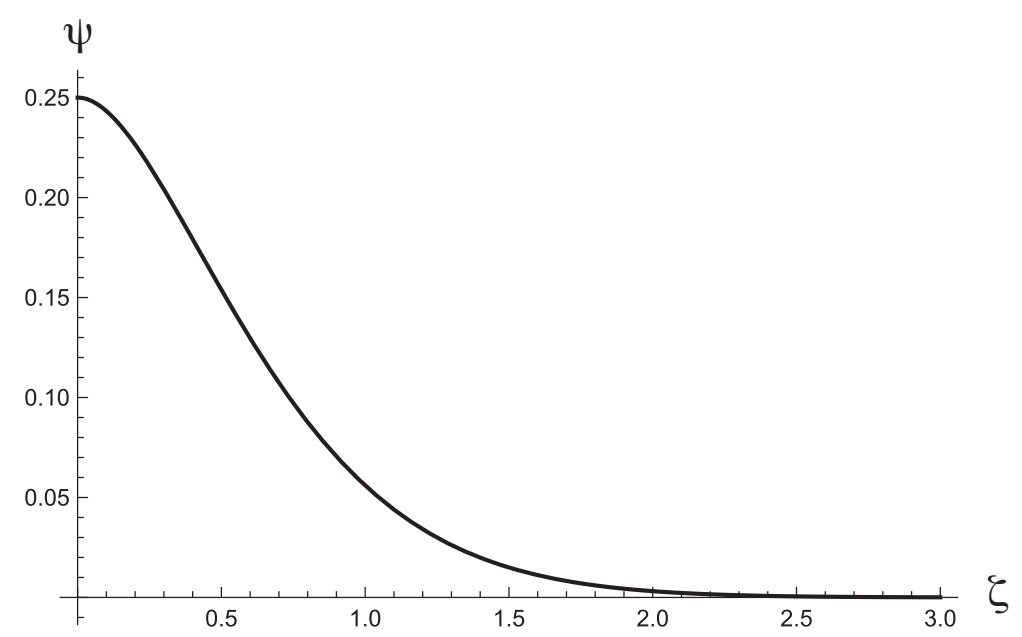

FIG. 5. Temperature profile in the thermal boundary layer for $\operatorname{Pr}=1$, from numerical solution of (4.4) with (5.1).

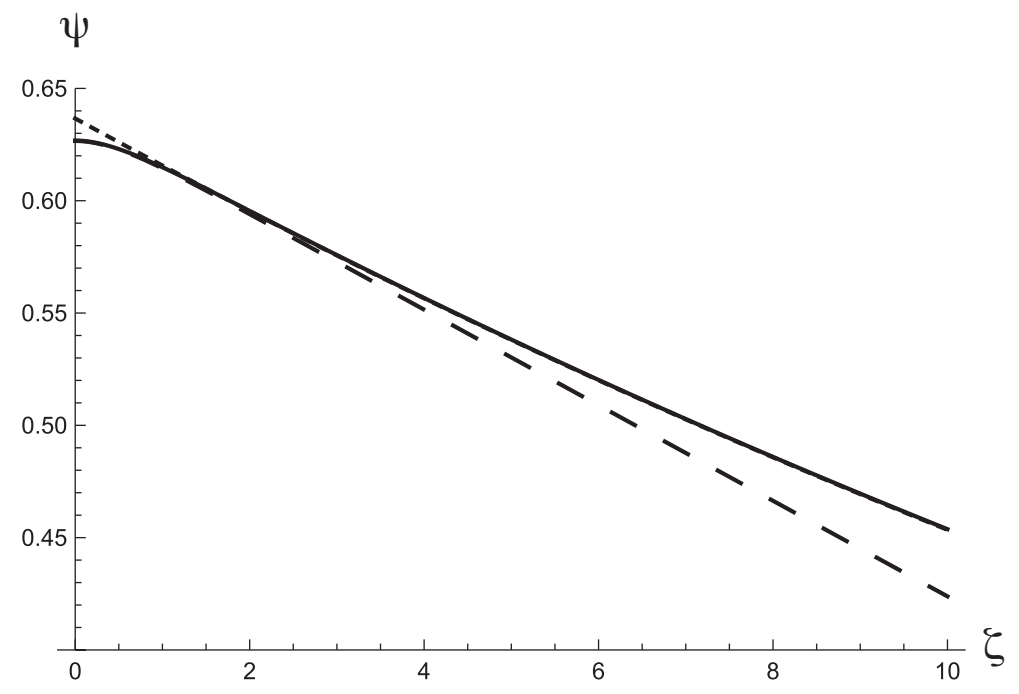

FIG. 6. Temperature profiles in the thermal boundary layer for $\operatorname{Pr}=10^{4}$ with adiabatic wall: numerical solution of (4.4) with (5.1) (solid line); inner solution (5.5) for large Pr (dashed line); outer (zero-conduction) solution from (4.10) (dotted line). 


\subsection{Boundary layer structure at small Prandtl number}

As in Section 4.2 above, the inner solution is obtained by ignoring terms of order Pr in (4.20), but with the adiabatic boundary condition we obtain

$$
\begin{aligned}
\phi=C+ & \frac{8 \sqrt{2}}{3 \sqrt{\pi}} \eta-\frac{16}{3 \sqrt{\pi}} \eta \operatorname{erf}(\eta)+\frac{1}{3}\left(3-\eta^{4}\right)\left(\operatorname{erfc}\left(\frac{\eta}{\sqrt{2}}\right)\right)^{2} \\
& +\frac{2 \sqrt{2}}{3 \sqrt{\pi}} \eta\left(\eta^{2}-1\right) e^{-\eta^{2} / 2} \operatorname{erfc}\left(\frac{\eta}{\sqrt{2}}\right)-\frac{2}{3 \pi}\left(6+\eta^{2}\right) e^{-\eta^{2}}
\end{aligned}
$$

from which

$$
\psi=\operatorname{Pr} \phi \sim \operatorname{Pr}\left(C+\frac{8 \sqrt{2}-16}{3 \sqrt{\pi}} \eta\right) \quad \text { as } \eta \rightarrow \infty .
$$

The outer solution is again (4.22), so the matching procedure equates (4.24) to (5.8) to yield

$$
A=\frac{\sqrt{2}-1}{3} \operatorname{Pr}^{1 / 2}, \quad C=(\sqrt{2}-1) \operatorname{Pr}^{-1 / 2} .
$$

From the inner solution (5.7) we find the wall temperature to be

$$
\psi(0)=\operatorname{Pr}^{1 / 2}(\sqrt{2}-1)+\operatorname{Pr}\left(1-\frac{4}{\pi}\right),
$$

which agrees with the result from numerical solution of (4.4) with (5.1) to within $0.5 \%$ at $\operatorname{Pr}=0.01$. This numerical solution is plotted together with the inner and outer asymptotic solutions in Figure 7.

\section{Physical scales}

We have considered the initial development of the flow and temperature profile in a channel of halfwidth $L$ when a constant pressure gradient is imposed from time $t=0$ on initially static, isothermal fluid. Away from the channel walls, the fluid has a uniform, constant acceleration

$$
A=\frac{1}{\rho_{m}} \frac{\mathrm{d} P_{d}}{\mathrm{~d} Z}
$$

and remains at its initial temperature $T_{0}$. Momentum and thermal boundary layers appear at the walls, and grow diffusively so that their thicknesses at time $t$ are, respectively,

$$
\delta_{m} \sim\left(\frac{\mu t}{\rho_{m}}\right)^{1 / 2}, \quad \delta_{\theta} \sim \operatorname{Pr}^{-1 / 2} \delta_{m}
$$

This formulation of uniform outer flow with boundary layers remains valid as long as both boundary layers remain thin compared to the channel half-width, i.e. at times less than $t_{b m}=\rho_{m} L^{2} / \mu$ if the Prandtl number is large or of order unity, or less than $t_{b \theta} \equiv \rho_{m} c L^{2} / k$ if the Prandtl number is small; note that $t_{b \theta}$ is the scale that was used to nondimensionalise time in (2.5). With $t$ in seconds and $L$ in metres, water $(\operatorname{Pr} \approx 7)$ has $t_{b m} \approx 10^{6} L^{2}$ whereas in glycerol $\left(\operatorname{Pr} \approx 1.3 \times 10^{4}\right), t_{b m} \approx 8 \times 10^{2} L^{2}$; in mercury $(\operatorname{Pr} \approx 0.027)$, $t_{b \theta} \approx 2 \times 10^{5} L^{2}$. [Here and below, values of physical parameters for liquids are those at $20^{\circ} \mathrm{C}$ according 


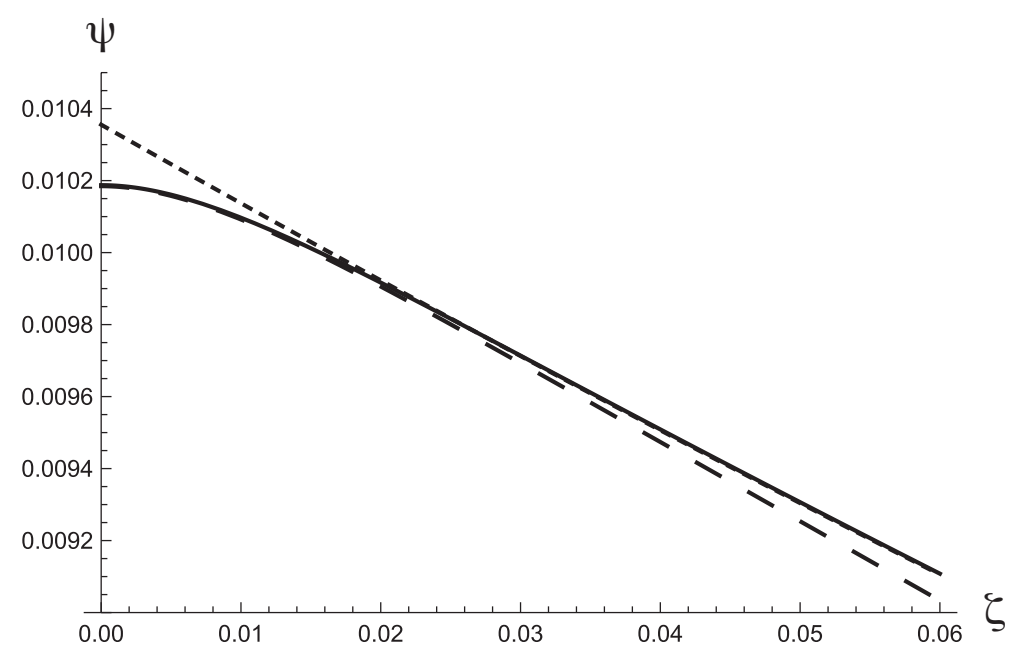

FIG. 7. Temperature profiles in the thermal boundary layer for $\operatorname{Pr}=6.25 \times 10^{-4}$ with adiabatic wall: numerical solution of (4.4) with (5.1) (solid line); inner solution $\psi=\operatorname{Pr} \phi$ with $\phi$ from (5.7) (dashed line) and outer solution (4.22) (dotted line), with constants given by (5.9).

to http://www.freestudy.co.uk/liquid\%20properties.htm (retrieved 16 August 2017), and should only be taken as approximate representative values, especially since some of the parameters vary considerably with temperature.]

At times $t \lesssim t_{b m}$ the fluid outside the boundary layers will have accelerated to a speed $V=A t$ which is independent of fluid properties apart from density, although the maximum speed attainable is given by the Poiseuille velocity scale

$$
A t_{b m} \sim \frac{L^{2}}{\mu} \frac{\mathrm{d} P_{d}}{\mathrm{~d} Z}
$$

The rather opaque scalings (2.6), (2.7) and (4.2) together with (6.1) and $V=A t$ resolve to the simple relation

$$
T-T_{0}=\frac{V^{2}}{c} \psi
$$

which embodies the idea that the temperature rise is due to conversion of kinetic energy to heat. Our solutions for large Prandtl number have shown that $\psi$ is bounded above by $2 / \pi$ (for both isothermal and adiabatic wall conditions), so that even if glycerol was accelerated to $10 \mathrm{~m} \cdot \mathrm{s}^{-1}$ it would experience a temperature rise of less than $0.03^{\circ} \mathrm{C}$. With small Prandtl number we have found that $\psi<\operatorname{Pr}^{1 / 2}(\sqrt{2}-1)$ with an adiabatic wall, and $\psi$ is even smaller $(\propto \operatorname{Pr})$ with an isothermal wall. Liquid metals tend to have low specific heat capacities, which somewhat compensates for the effects of small Prandtl number in terms of temperature rise according to (6.4); however, mercury accelerated to $10 \mathrm{~m} . \mathrm{s}^{-1}$ with an adiabatic wall would still experience a temperature rise of less than $0.05^{\circ} \mathrm{C}$.

The total heat generated per unit mass of fluid is

$$
V^{2} \sqrt{2 \tau} \int_{0}^{\infty} \psi \mathrm{d} \zeta \approx 0.25 V^{2}\left(\operatorname{Pr} \frac{t}{t_{b \theta}}\right)^{1 / 2}
$$


where the factor $\sqrt{2}$ on the left arises from our scalings, and the right-hand estimate applies according to (5.2) when the walls are adiabatic so that no heat can escape. With $\operatorname{Pr} \lesssim 1$ the accelerating phase of flow has ended by the time $t / t_{b \theta}=1$, but if the Prandtl number is large it ends when $t / t_{b \theta} \sim \operatorname{Pr}^{-1}$. Thus, even with glycerol, the total heat generated in the accelerating phase if the fluid has a final velocity of $10 \mathrm{~m} . \mathrm{s}^{-1}$ is only about $25 \mathrm{~J} . \mathrm{kg}^{-1}$.

If the walls are isothermal, there is heat flux

$$
\left.k \frac{\partial T}{\partial Y}\right|_{\text {wall }}=\left.\left(\frac{k \rho_{m}}{2 c}\right)^{1 / 2} V^{3 / 2} A^{1 / 2} \frac{\mathrm{d} \psi}{\mathrm{d} \zeta}\right|_{\zeta=0}
$$

through the walls when the flow has reached velocity $V$ at acceleration $A$. Values of $\mathrm{d} \psi /\left.\mathrm{d} \zeta\right|_{\zeta=0}$ are less than 1.4 for large Prandtl number, and less than $0.9 \operatorname{Pr}^{1 / 2}$ for small Prandtl number, according to (4.17) and (4.26) respectively. If we take the rather extreme situation of an applied pressure gradient equal to the hydrostatic value, so that the fluid accelerates at $g$ to reach $V=10 \mathrm{~m} \cdot \mathrm{s}^{-1}$ in 1 second, the wall heat flux with glycerol would still be less than $40 \mathrm{~W} \cdot \mathrm{m}^{-2}$.

We neglected the buoyancy term in (2.8) on the basis that $\theta=O\left(\tau^{2}\right)$ (and $\theta_{m}=O\left(\tau^{5 / 2}\right)$ ) when we solved the system (2.8) and (2.9) by formal expansion in powers of $\tau$. In terms of physical magnitude, the buoyancy may be compared with the applied pressure gradient:

$$
\frac{\theta}{\Pi}=\frac{g \beta}{c} A t^{2} \psi
$$

where $g \beta / c \approx 1.3 \times 10^{-5} \mathrm{~m}^{-1}$ for mercury (and has smaller values for water and glycerol). Since $\psi=O(1)$ (or smaller) in all our solutions, it is clear that the buoyancy force resulting from viscous dissipation in the acceleration phase is unlikely to be significant, at least in flows on the laboratory or industrial scale. The quantity $(g \beta / c) A t^{2}$ in (6.7) is essentially the same as the local dissipation number introduced by Gebhart (1962) as a measure of the contribution of viscous dissipation in steady free-convection flow adjacent to a heated wall, and this author comes to similar conclusions about the importance of viscous heating in engineering applications. Furthermore, proceeding to $O\left(\tau^{2}\right)$ in (2.8) to determine the effect of this buoyancy force on the flow seems unlikely to be of great mathematical interest, so we have not pursued this. Thus the original specification that the channel is vertical becomes irrelevant: with buoyancy force neglected, our equations apply equally to a channel in any orientation.

\section{Conclusions}

Our solutions apply to the initial accelerating stage of flow due to the sudden imposition of a pressure gradient in a channel, before Poiseuille flow becomes established, but also more generally to the boundary layer on a plane wall in a flow with constant acceleration. Because we have not examined the effects of the buoyancy force which results from viscous heating, the results are independent of the orientation of the channel or wall. The physical scales of the effects of dissipation in these accelerating flows have been shown to be rather small in most engineering applications, but the results do have some mathematical interest, particularly because most previous studies involving viscous dissipation have considered steady flows. We envisage that our solutions will be of use in initiating the numerical solution of heat transfer problems in flows started from rest.

Our analysis has solved the thermal boundary layer equation, in which the viscous dissipation term has been calculated from the velocity profile obtained by Blasius (1908) for the momentum boundary layer in an accelerating flow. We have paid particular attention to the asymptotic limits of large and small 
Prandtl number, and considered both isothermal and adiabatic wall conditions. Large Pr means that viscous dissipation occurs in a broad momentum boundary layer, within which the temperature adjusts to the wall condition in a narrower thermal layer. Thus the maximum temperature rise is approximately independent of the wall condition and of the Prandtl number for $\operatorname{Pr} \gg 1$, being given by (6.4) with $\psi \approx 2 / \pi$. In contrast, small Prandtl number means that viscous heating only occurs in a narrow layer, but the heat is conducted outwards to a broader thermal layer. Since the region where heat is generated becomes smaller as Pr decreases, the temperature rise also decreases with smaller Pr; furthermore, an isothermal wall allows heat to be removed from the fluid, so the reduction in temperature rise with decreasing $\operatorname{Pr}$ is more rapid $\left(T-T_{0} \propto \operatorname{Pr}\right)$ in this case than with an adiabatic wall $\left(T-T_{0} \propto \operatorname{Pr}^{1 / 2}\right)$.

\section{REFERENCES}

Barletta, A. (1998) Laminar mixed convection with viscous dissipation in a vertical channel. Int. J. Heat and Mass Transfer, 41, 3501-3513.

Barletta, A. (2008) Comments on a paradox of viscous dissipation and its relation to the Oberbeck-Boussinesq approach. Int. J. Heat and Mass Transfer, 51, 6312-6316.

Barletta, A. (2009) Local energy balance, specific heats and the Oberbeck-Boussinesq approximation. Int. J. Heat and Mass Transfer, 52, 5266-5270.

Barletta, A. (2015) On the thermal instability induced by viscous dissipation. Int. J. Thermal Sci., 88, $238-247$.

Barletta, A., Magyari, E. \& Keller, B. (2005) Dual mixed convection flows in a vertical channel. Int. J. Heat and Mass Transfer, 48, 4835-4845.

Barletta, A. \& Zanchini, E. (1999) On the choice of reference temperature for fully-developed mixed convection in a vertical channel. Int. J. Heat and Mass Transfer, 42, 3169-3181.

Blasius, H. (1908) Grenzschichten in Flüssigkeiten mit kleiner Reibung. Z. Math. Physik, 56, 1-37. English translation in NACA Technical Memorandum 1256, http://naca.central.cranfield.ac.uk/reports/1950/naca-tm1256.pdf.

Gebhart, B. (1962) Effects of viscous dissipation in natural convection. J. Fluid Mech., 14, 225-232.

Kay, A. (2016) When is natural convection completely passive? Z. Angew. Math. Mech., 96, 279-303.

Lamb, H. (1927) A paradox in fluid motion. J London Math Soc, 2, 109-112.

Miklavčič, M. (2015) Stability analysis of some fully developed mixed convection flows in a vertical channel. $Z$. Angew. Math. Mech., 95, 982-986.

Miklavčič, M. \& Wang, C.Y. (2011) Completely passive natural convection. Z. Angew. Math. Mech., 91, 601-606.

Turcotte, D.L., Spence, D.A. \& Bau, H.H. (1982) Multiple solutions for natural convective flows in an internally heated, vertical channel with viscous dissipation and pressure work. Int. J. Heat and Mass Transfer, 25, 699706. 